\title{
Are stirring and sonication pre-dispersion methods equivalent for in vitro toxicology evaluation of $\mathrm{SiC}$ and $\mathrm{TiC}$ ?
}

\author{
Jorge Mejia Vanessa Valembois · Jean-Pascal Piret • Frans Tichelaar • \\ Marijn van Huis · Bernard Masereel · Olivier Toussaint · Joseph Delhalle • \\ Zineb Mekhalif · Stéphane Lucas
}

Received: 3 November 2011/Accepted: 9 March 2012

(C) Springer Science+Business Media B.V. 2012

\begin{abstract}
The evolution of the particle size distribution and the surface composition of silicon carbide and titanium carbide nanoparticle (NP) dispersions were studied. The pre-dispersions were prepared using two commonly used protocols for dispersion: stirring
\end{abstract}

Electronic supplementary material The online version of this article (doi:10.1007/s11051-012-0815-7) contains supplementary material, which is available to authorized users.

J. Mejia $(\bowtie) \cdot$ V. Valembois $\cdot$ S. Lucas

Research Centre for the Physics of Matter and Radiation (LARN-PMR), NARILIS, University of Namur-FUNDP, Rue de Bruxelles 61, 5000 Namur, Belgium

e-mail: jorge.mejiamendoza@fundp.ac.be

J.-P. Piret · O. Toussaint

Research Unit in Cellular Biology (URBC), NARILIS, University of Namur-FUNDP, Rue de Bruxelles 61, 5000 Namur, Belgium

F. Tichelaar · M. van Huis

National Centre for HRTEM, Kavli Institute

of Nanoscience, Delft University of Technology,

Lorentzweg 1, 2628 CJ Delft, The Netherlands

B. Masereel

Department of Pharmacy NAMEDIC, Namur Thrombosis and Hemostasis Center (NTHC), University

of Namur-FUNDP, Rue de Bruxelles 61,

5000 Namur, Belgium

J. Delhalle $\cdot$ Z. Mekhalif

Laboratory of Chemistry and Electrochemistry

of Surfaces-CES, University of Namur-FUNDP, Rue de

Bruxelles 61, 5000 Namur, Belgium and sonication. Two dispersants were investigated (water and Pluronic F108 1\%) at two stages: predispersion and during in vitro assays. Our data show that for each tested condition, different time-dependent results for the surface chemical composition as well as size and percentage of the agglomerates and the primary particles are observed. De-agglomeration and successive or simultaneous cleaning-wrapping cycles of the nanomaterial are observed and are related to the dispersion method and the medium as well as to the chemical stability of the NP surface. Biological response during in vitro assessment was also performed for one given pre-dispersion time condition and demonstrates that the preparation method significantly alters the results.

Keywords SiC nanoparticles · TiC nanoparticles · Particle size distribution - Surface composition .

Protocol of dispersion - In vitro assays .

Effective dose $\cdot$ Environmental and health effects

\section{Introduction}

Silicon carbide $(\mathrm{SiC})$ is a unique ceramic material produced by different methods, including but not limited to carbothermal reduction, sol-gel method, gas-phase reaction method, self-propagation hightemperature synthesis, and plasma production (Lin et al. 2008). It has a wide spectrum of uses due to its excellent mechanical and chemical stability at 
elevated temperatures (Fukushima et al. 2008). It is considered to be a highly biocompatible material with promising uses in bioanalysis, biotechnology, medicine, surface coating of devices intended for contact with tissues, and as film for serum protein adsorption or cell culturing (Barillet et al. 2010; Guevara-Lora et al. 2009). Titanium carbide ( $\mathrm{TiC}$ ) nanoparticles (NPs) can be produced by similar methods as those used for SiC NPs; they are a technologically important material for crucibles, cutting tools, polishing pastes, transparent optical materials, armor, and composites for tape heads in the electronic and wear resistant materials industry (Bae et al. 2009).

These characteristics have driven increased production of $\mathrm{SiC}$ and TiC NPs. Even if their popularity has grown, concerns about their potential risk have also been raised (Nel et al. 2006). Consequently, their potential toxicity has been the subject of attention in recent years (Borm et al. 2006; Sager et al. 2007), and a thorough physicochemical characterization is essential to understand and assess the risks (Hoet et al. 2004).

The choice of adequate parameters to follow the modifications induced by the dispersion in aqueous media is crucial. The particle size distribution (PSD) and the surface composition of the NPs are among the most important parameters governing their behavior in any given dispersion media (Karakoti et al. 2006). Proper tuning can improve the degree of dispersion (Iijima and Kamiya 2008; Novak et al. 2007; Vauthier et al. 2008) and reduce agglomeration (Vauthier et al. 2008; Yates et al. 2008).

The method used for the preparation of the NP dispersions (stirring, sonication, centrifugation, etc.) is a parameter by itself (Bihari et al. 2008; Che et al. 2007; Foucaud et al. 2007), since the energy provided may modify the size and composition of pristine nanomaterials. To date, however, the evolution of the PSD and the surface composition from carbide NPs dispersions, prepared by different protocols of dispersion, has not been studied. That is the purpose of this study.

We present here the detailed characterization for $\mathrm{SiC}$ and TiC NPs, before and after dispersion in water and in a Pluronic F108 solution. Pluronic F108 is a non-ionic triblock copolymer of polyethylene oxide/ polypropylene oxide/polyethylene oxide (PEO/PPO/ PEO) often used for toxicology assessment tests (Cherukuri et al. 2004, 2006; Corey et al. 2009). Two dispersion methods typically used in the toxicity assessment of NPs are studied: stirring and sonication. Their impact on cell viability or interleukin-8 (IL-8) release by immortalized human N-hTERT keratinocytes has also been investigated. These keratinocytes express telomerase and lack a functional cyclindependent kinase inhibitor p16INK4a, which renders them immortal while retaining their full differentiation potential (Dickson et al. 2000).

\section{Methods}

Materials

$\mathrm{SiC}$ and TiC NPs were purchased from Ionic Liquids Technologies $\mathrm{GmbH} \&$ Co KG, Germany (Iolitec 2007a, b) and employed as received. Pluronic F108, a non-ionic surfactant (PEO/PPO/PEO, chemical formula: $\mathrm{HO}\left(\mathrm{C}_{2} \mathrm{H}_{4} \mathrm{O}\right)_{a}\left(-\mathrm{C}_{3} \mathrm{H}_{6} \mathrm{O}\right)_{b}\left(\mathrm{C}_{2} \mathrm{H}_{4} \mathrm{O}\right)_{a} \mathrm{H}$ with $a=$ 129 and $b=56, \mathrm{Mw}=14,600)$, was purchased from BASF Inc. (Germany).

Dispersion methods

Two types of NP dispersions were prepared at a concentration of $1 \mathrm{mg} / \mathrm{mL}$ : dispersed in water and dispersed in $1 \%$ w/v Pluronic F108 aqueous solution, according to two distinct protocols of dispersion: stirring and sonication probe (Mejia et al. 2011; Piret et al. 2010; Vankoningsloo et al. 2010). The water dispersion was prepared by adding the NPs directly to water at room temperature. The $1 \% \mathrm{w} / \mathrm{v}$ Pluronic F108 aqueous solution was prepared by dissolving pellets of Pluronic F108 in water at room temperature by magnetic stirring for $30 \mathrm{~min}$. All solutions were prepared with ultrapure water (milliQ $18.2 \mathrm{M} \Omega \mathrm{cm}$ ).

The effects of the two dispersion methods were compared at $10 \mathrm{~min}$ intervals, from 0 to $300 \mathrm{~min}$ on two independent set of samples. This time span was selected since most of the protocols of dispersion use the method of dispersion for quite a bit less time (between 10 and 120 min) (Piret et al. 2010; Vankoningsloo et al. 2010):

Method 1: stirring (600 rpm, IKA Werke RCT Basic). Measured power input was negligible. Method 2: sonication (Vibracell VC50-T $20 \mathrm{kHz}$ $50 \mathrm{~W}$, Analis, regulated at $6.25 \mathrm{~W}$ ). Measured power input was $0.62 \mathrm{~W}$. 
The 5-mL vial containing samples from sonication were placed in an ice water bath to prevent any temperature increase. The power input was estimated from the power transfer equation (Raman and Abbas 2008):

$P_{\text {cal }}=m_{\mathrm{L}} C_{P}\left(\frac{\partial T}{\partial t}\right)_{t=0}$

$P_{\text {cal }}$ is the calorimetrically determined power (W), $C_{P}$ is the specific heat capacity of liquid $\left(4.186 \mathrm{~kJ} / \mathrm{kg}{ }^{\circ} \mathrm{C}\right.$ for water at $15{ }^{\circ} \mathrm{C}$ and assumed constant), and ( $\partial T /$ $\partial t)_{t=0}$ is the rate of change of the uncontrolled system temperature at the start of sonication.

\section{Characterization methods}

The morphology and structure of the SiC NPs were characterized using a high-resolution transmission electron microscope (HRTEM) at an acceleration voltage of $200 \mathrm{kV}$ (Monochromated Tecnai 2001, Philips) and a field emission gun electron microscope Jeol JSM-7500F (FEG-SEM) equipped with an energy dispersive X-ray detector (operating at $15 \mathrm{kV}$, working distance of $8 \mathrm{~mm}$ and with a resolution of $1.0 \mathrm{~nm}$ at $15 \mathrm{kV}$ ). The specific surface area was determined by the BET method (ASAP 2010, Micromeritics), and the elemental chemical composition was analyzed by EDX at $20 \mathrm{keV}$ (analysis station JED 2300 detector coupled to JSM-7500F, ZAF corrections). The estimated depth analysis in $\mathrm{SiC}$ and TiC NPs is about $10 \mu \mathrm{m}$.

X-ray photoelectron spectroscopy (XPS) was used for qualitative and quantitative elemental analysis of the surface, with a SSX-100 spectrometer using the monochromatized X-ray $\mathrm{Al} \mathrm{K}_{\alpha}$ radiation, $1486.6 \mathrm{eV}$. The spectra were recorded at a $35^{\circ}$ take-off angle. The analyzed core-level lines were calibrated with respect to the $\mathrm{C} 1 s$ peak at $284.6 \mathrm{eV}$ and to the $\mathrm{Au} 4 f_{7 / 2}$ $(84.0 \mathrm{eV})$ from the substrate surface used for placing the samples. The spectra were analyzed, fitting the Gaussian function to the experimental curve, with a nonlinear least squares scheme. Nominal resolution was measured as full width at half maximum of $1.0 \mathrm{eV}$ (core-level spectrum) to $1.5 \mathrm{eV}$ (survey spectrum). The estimated depth analysis in SiC and TiC NPs is about $5 \mathrm{~nm}$. Ions released were measured by atomic absorption spectroscopy (AAS) with a Philips PU 9200X flame spectrometer from centrifuged NP dispersions. Zeta potential was measured, without sample dilution on the prepared dispersions, with a Delsa $^{\text {TM }}$ Nano C equipped with a laser diode, $658 \mathrm{~nm}$ $(30 \mathrm{~mW})$ and measured with a high-concentration cell at a scattering angle of $30^{\circ}$.

PSD of the dispersed NPs was measured with a disc centrifuge CPS 24,000 (CPS instruments Inc., USA) working at $24,000 \mathrm{rpm}$. The disc centrifuge measurement is based on centrifugal liquid sedimentation (CLS) according to Stokes' law (Mejia et al. 2011; Merkus 2009). The accuracy of measured sizes is ensured before each test through the use of a certified size calibration standard (particle size: $226 \mathrm{~nm}$ ). The laser of the disc centrifuge used to measure the extinction has a wavelength of $\lambda=405 \mathrm{~nm}$ associated with a refractive index of 2.76 for SiC NPs and 2.75 for TiC NPs. For the specific measurements in Pluronic F108 dispersions, a sucrose gradient was prepared with the same $1 \%$ w/v Pluronic F108 aqueous solution. In the case of $\mathrm{SiC}$ and TiC NPs dispersed in water, the sucrose gradient was prepared with ultrapure water.

\section{Cell culture and incubation}

Human telomerase-immortalized keratinocytes (NhTERT) cells (Dickson et al. 2000) were kind gifts from Dr. J.G. Rheinwald (Department of Medicine and Harvard Skin Disease Research Center). Cells were maintained in Epilife medium with human keratinocyte growth supplement (Cascade Biologics) and antibiotics (penicillin/streptomycin). Cells were grown in a $5 \% \mathrm{CO}_{2}$ incubator in humidified atmosphere at $37^{\circ} \mathrm{C}$.

\section{Biological effects}

To evaluate the biological impact of $\mathrm{SiC}$ and TiC NPs, N-hTERT keratinocytes were incubated for 24 and $48 \mathrm{~h}$ either with or without (control, CTL) $100 \mu \mathrm{g} / \mathrm{mL}$ of NPs. Controls for pluronic-dispersed $\mathrm{SiC}$ and $\mathrm{TiC}$ NPs were made in the presence of pluronic solutions at the same dilutions. Controls and tests for watersuspended $\mathrm{SiC}$ and TiC NPs received an equivalent volume of water. Benzalkonium chloride (BC) (Sigma) was used at $10 \mu \mathrm{g} / \mathrm{mL}$ as positive control (Vankoningsloo et al. 2010).

Cytotoxic and pro-inflammatory effects of $\mathrm{SiC}$ and TiC NPs were assayed by quantifying the amount of lactate dehydrogenase released from cells with 
impaired plasma membranes and the pro-inflammatory IL-8 released in culture medium, respectively.

The release of LDH outside damaged cells was assessed with the Cytotoxicity Detection kit (Roche) according to manual instructions. Briefly, culture supernatants were collected and mixed with LDH detection reactants for $10 \mathrm{~min}$ before measuring at $490 \mathrm{~nm}$ absorbance with a spectrophotometer (xMark, Biorad) and normalizing values for protein contents with Pierce $660 \mathrm{~nm}$ Protein Assay Reagent (Thermo). The concentration of IL-8 in culture medium was assayed by specific sandwich ELISA (Quantikine human IL-8 Immunoassay, R\&D Systems) according to the manufacturer's protocol. IL- 8 values were normalized with protein contents (Pierce $660 \mathrm{~nm}$ Protein Assay Reagent, Thermo Scientific).

The anti-proliferative molecules used as positive controls in preliminary optimization tests were $1 \mathrm{mg} /$ $\mathrm{mL} \mathrm{BC}$, applied topically, or a combination of $10 \mathrm{ng} /$ $\mathrm{mL}$ tumor necrosis factor $\alpha$ (TNF $\alpha)$ (Sigma) and interferon $\gamma$ (IFN $\gamma)$ (Sigma), diluted in the culture medium.

\section{Sample preparation for characterization}

The grids used for HRTEM observation were made of holey carbon film on a Cu 200 mesh grid (Quantifoil R1.2/1.3). Samples from dry pristine materials were placed on double-sided conductive tape for XPS analysis. Liquid dispersion samples for XPS and EDX were placed on a gold substrate $(100 \mathrm{~nm}$ thick) and analyzed at room temperature. All samples from the pluronic dispersions were filtrated under vacuum with a $0.2-\mu \mathrm{m}$ pore size membrane (Anodisc 25,
Whatman) and redispersed in water several times to remove, by centrifugation cycles $(3,000 \mathrm{rpm}$ for $5 \mathrm{~min}$ ), non-adsorbed species. Samples from the in vitro assessment were obtained as follows: NPs suspensions were first diluted at $100 \mu \mathrm{g} / \mathrm{mL}$ in culture medium.

After 6, 24, 40, or $48 \mathrm{~h}$ of incubation, the different portions were collected as follows: (a) the culturemedium portion (CMP) was collected directly after the incubation time and (b) the cell portion (CP) corresponds to the difference between the whole and the medium portion, recovered after incubation. Later, one drop of the sample was placed on a gold substrate for XPS analyses. For the PSD analysis, $0.2 \mathrm{~mL}$ was used for each analysis. Samples were prepared and analyzed as soon as they were produced, and all experiments were conducted at room temperature. Samples for AAS were prepared from the supernatant of water dispersion after centrifugation of the NPs.

\section{Statistical analysis}

Results are expressed as mean \pm standard deviation (SD). Data were analyzed by Student's $t$ tests.

\section{Results}

Characterization of the pristine material

Table 1 contains the most important characterization results for the pristine $\mathrm{SiC}$ and TiC NPs as dry powder. EDX and XPS values correspond to atomic percentages.

Table 1 Characterization of the bulk and the surface of pristine $\mathrm{SiC}$ and TiC NPs

\begin{tabular}{|c|c|c|c|c|c|c|c|c|c|c|c|c|}
\hline & \multicolumn{6}{|l|}{$\mathrm{SiC}$} & \multicolumn{6}{|l|}{$\mathrm{TiC}$} \\
\hline & \multicolumn{3}{|c|}{ Measured } & \multicolumn{3}{|c|}{ Supplier ${ }^{\mathrm{a}}$} & \multicolumn{3}{|c|}{ Measured } & \multicolumn{3}{|c|}{ Supplier $^{\mathrm{a}}$} \\
\hline Average diameter (SEM), nm & \multicolumn{3}{|c|}{$53.4 \pm 12.4$} & \multicolumn{3}{|l|}{60} & \multicolumn{3}{|c|}{$39.6 \pm 8.9$} & \multicolumn{3}{|l|}{20} \\
\hline Surface area $\left(\mathrm{m}^{2} / \mathrm{g}\right)$ & \multicolumn{3}{|c|}{30} & \multicolumn{3}{|l|}{80} & \multicolumn{3}{|l|}{16} & \multicolumn{3}{|l|}{50} \\
\hline \multirow[t]{2}{*}{ Crystallography } & \multicolumn{3}{|c|}{$\beta-\mathrm{SiC}$} & \multicolumn{3}{|c|}{$\beta-\mathrm{SiC}$} & \multicolumn{3}{|c|}{ Cubic } & \multicolumn{3}{|l|}{-} \\
\hline & $\mathrm{Si}$ & $\mathrm{C}$ & $\mathrm{O}$ & $\mathrm{Si}$ & $\mathrm{C}$ & $\mathrm{O}$ & $\mathrm{Ti}$ & $\mathrm{C}$ & $\mathrm{O}$ & $\mathrm{Ti}$ & $\mathrm{C}$ & $\mathrm{O}$ \\
\hline Bulk composition (EDX) & 26.9 & 65.5 & 7.5 & \multirow{2}{*}{\multicolumn{3}{|c|}{$99.0^{\mathrm{b}}$}} & 36.9 & 59.5 & 3.6 & \multirow{2}{*}{\multicolumn{2}{|c|}{$99.0^{\mathrm{c}}$}} & \\
\hline Surface composition (XPS) & 36.9 & 26.9 & 36.2 & & & & 8.1 & 64.5 & 27.4 & & & \\
\hline
\end{tabular}

\footnotetext{
${ }^{\text {a }}$ Iolitec technical data sheet 05/01/2007

b Product purity reported as SiC only

c Product purity reported as TiC only
} 
One can see that the bulk chemical composition is different from the supplier's datasheet and is remarkably different from the surface composition. This is mainly due to the presence of $\mathrm{O}$ at the surface in excess of 36.2 and $27.4 \%$ for $\mathrm{SiC}$ and $\mathrm{TiC}$, respectively. Also, the $\mathrm{C}$ content of the NPs surface is not related to the perfect $\mathrm{TiC}$ or $\mathrm{SiC}$ chemical composition. Carbon is under stoichiometric for $\mathrm{SiC}$, while it is over stoichiometric for TiC. Buck composition as measured by EDX shows that both NPs exhibit an excess in C, and that $\mathrm{TiC}$ is the NPs closest to the supplier datasheet chemically speaking. From surface to volume, the ratio of metal (to facilitate the reading, $\mathrm{Si}$ and $\mathrm{Ti}$ are considered as metals) over $\mathrm{C}$ atomic concentration (Me/C) decreases from 1.37 to 0.41 for $\mathrm{SiC}$ and increases from 0.13 to 0.62 for $\mathrm{TiC}$.

Precise line shape and position analysis of the $\mathrm{Si}$ XPS region shows that the outer surface of $\mathrm{SiC}$ is composed of a mixture of $\mathrm{SiO}_{2}, \mathrm{SiC}$, and $\mathrm{Si}$ (see Supplemental data). Ti XPS region shows the presence of metallic $\mathrm{Ti}$ and $\mathrm{Ti}_{x} \mathrm{C}_{y} \mathrm{O}_{z}$, as already seen by Hassan et al. (2008).

Measurements from SEM images indicate that the SiC NPs have an average diameter of $53.4 \pm 12.4 \mathrm{~nm}$ and TiC NPs have an average diameter of $39.6 \pm$ $8.9 \mathrm{~nm}$. However, agglomeration of pristine material is observed as shown on HRTEM images in Fig. 1(1 and 4). This will be confirmed by CLS measurements (see beneath). Figure 1(3 and 6) shows the size distribution of pristine SiC and TiC NPs. Additional
X-ray diffraction experiments (data not shown) revealed that both materials have a crystalline signature and that crystal size, calculated from the Scherrer formula, are around 12.7 and $24.2 \mathrm{~nm}$ for $\mathrm{SiC}$ and $\mathrm{TiC}$ NPs, respectively. These values are significantly lower than the average diameter of the NPs as measured by HRTEM (Fig. 1(4 and 7)), suggesting that there is a crystalline core and an amorphous shell.

Data from the specific surface area points to a difference in the adsorbent character of the material with respect to the data provided 30 and $16 \mathrm{~m}^{2} \mathrm{~g}^{-1}$ for the $\mathrm{SiC}$ and $\mathrm{TiC}$, respectively.

Modifications of the pristine material

\section{During pre-dispersion}

Size distribution and primary particles (PPs) percentage Raw data of all the dispersions always exhibit two distinctive peaks: one located at a hydrodynamic diameter of around $25 \mathrm{~nm}(15 \mathrm{~nm}$ peak width at half height)_associated with small individual particles (Supplementary Fig. 1, available online); and a second peak at larger hydrodynamic diameters $(>200 \mathrm{~nm})$ due to the presence of agglomerates. Depending on the dispersion method and the power input, these agglomerates can deagglomerate and generate a time-dependent behavior. A variation in the averaged hydrodynamic diameter of the agglomerates and individual NPs can also be
Fig. 1 HRTEM images of pristine $\mathrm{SiC}$ and TiC NPs. Pristine SiC NPs agglomerated (1), image of SiC NPs (2), PSD of SiC NPs from HRTEM images (3), pristine TiC NPs agglomerated (4), image of TiC NPs (5), PSD of TiC NPs from HRTEM images (6). Scale bars correspond to $200 \mathrm{~nm}(\mathbf{1}), 10 \mathrm{~nm}(\mathbf{2}, \mathbf{5})$, and $50 \mathrm{~nm}$ (4)
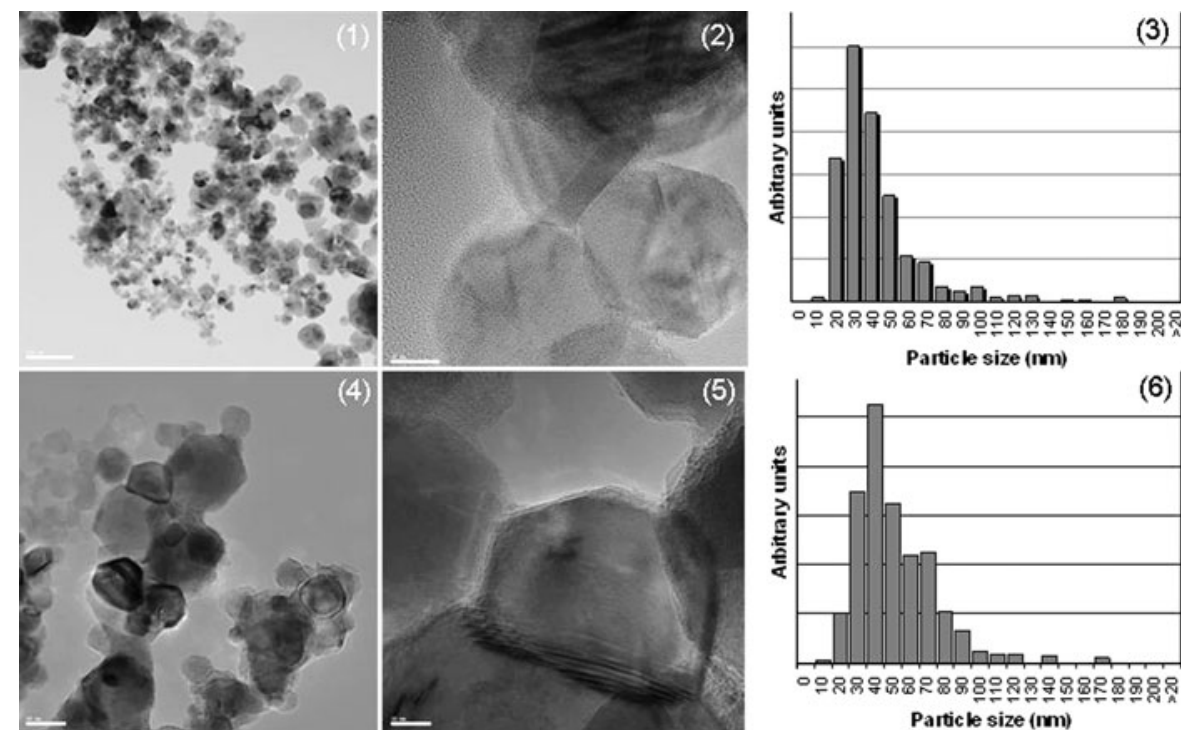
observed. For this reason, we chose to divide the data into two size distributions: those smaller than $90 \mathrm{~nm}$ have been called PPs, while those larger than $90 \mathrm{~nm}$ are considered to be agglomerates. The arbitrary cutoff at a hydrodynamic diameter of $90 \mathrm{~nm}$ represents the lowest intensity point that divides the PSD in the two aforementioned size distributions (Supplementary Figs. 1 and 2, available online). This definition is in agreement with the one proposed by Taurozzi et al. (2011).

Figure 2 shows the evolution of the average hydrodynamic diameter size of the PPs and the agglomerates of $\mathrm{SiC}$ and $\mathrm{TiC} \mathrm{NP}$ dispersions by stirring and sonication, in water and pluronic dispersions.

Generally speaking, stirring affects the average size of both the agglomerates and the PP less than sonication, regardless of the dispersion media. Indeed, if one reads Fig. 2 vertically (by dispersion technique), stirring has basically no influence on the $\mathrm{SiC} \&$ TiC PPs size, whatever the medium, over a 300-min time span. On the contrary, sonication systematically decreases the size of the $\mathrm{SiC}$ and $\mathrm{TiC}$ agglomerates while keeping constant the size of the PPs, whatever the medium. SiC agglomerates and PPs behave the same way as $\mathrm{TiC}$ except in the case of $\mathrm{TiC}$-water where a triggered de-agglomeration effect seems to be observed for sonication.
Figure 3 presents the percentage of PPs in the two media and with the two methods as measured by CLS. The percentage of PPs was obtained from PSD as the percentage of the area below the curve between 0 and $90 \mathrm{~nm}$ (see Supplementary Figs. 1 and 2, available online).

It is seen that the two dispersion methods and the chemical nature of the NPs significantly alter the amount of PPs percentage in a time-dependent way. Indeed, stirring in water promotes de-agglomeration of SiC NPs as the concentration of PPs increases linearly to reach $100 \%$ after $90 \mathrm{~min}$. On the contrary, stirring promotes re-agglomeration of $\mathrm{SiC}$ in pluronic, as the overall PPs percentage is reduced from 85 to $60 \%$ after $150 \mathrm{~min}$. The final percentage of stirred TiC PPs compared to the original one is less affected by the dispersion medium compared to SiC. A small increase of TiC PPs percentage in water (75-90 \%) and an unchanged concentration of about $80 \%$ in pluronic are observed after a cyclic variation of PPs percentage within the considered time span.

Sonication induces large variations in the SiC PPs percentage in water, finally allowing it to achieve a lower value. Cyclic variations of $\mathrm{SiC}$ PPs percentages are also observed in pluronic over the 300 min time span before finally reaching a reduced amount of $\mathrm{SiC}$ PPs percentage. The PPs percentage of TiC NPs varies smoothly when dispersed in either pluronic or water in
Fig. 2 Hydrodynamic diameter size of PPs and agglomerates in water (closed square) and in pluronic (closed triangle) dispersions obtained from CLS measurements. SiC NPs: stirring (1) and sonication (2). TiC NPs: stirring (3) and sonication (4). Trend lines are intended to guide the eyes of the reader

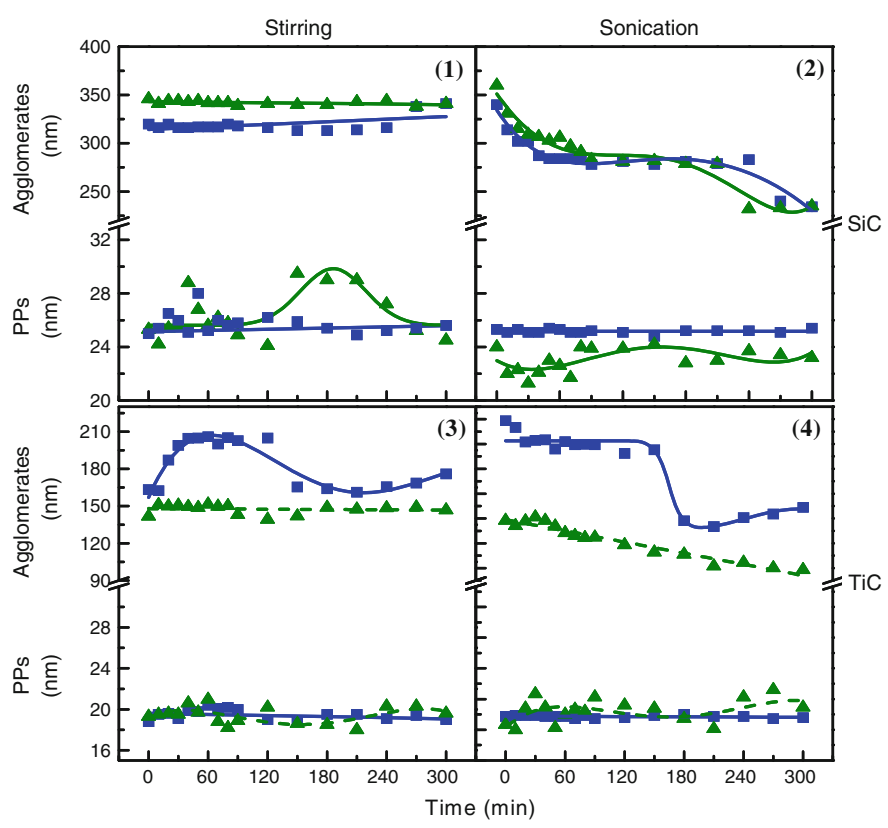


Fig. 3 PPs percentage from PSD in water (closed square) and in pluronic (closed triangle) dispersions obtained from CLS measurements. SiC NPs: stirring (1) and sonication (2). TiC NPs: stirring (3) and sonication (4). Trend lines are intended to guide the eyes of the reader

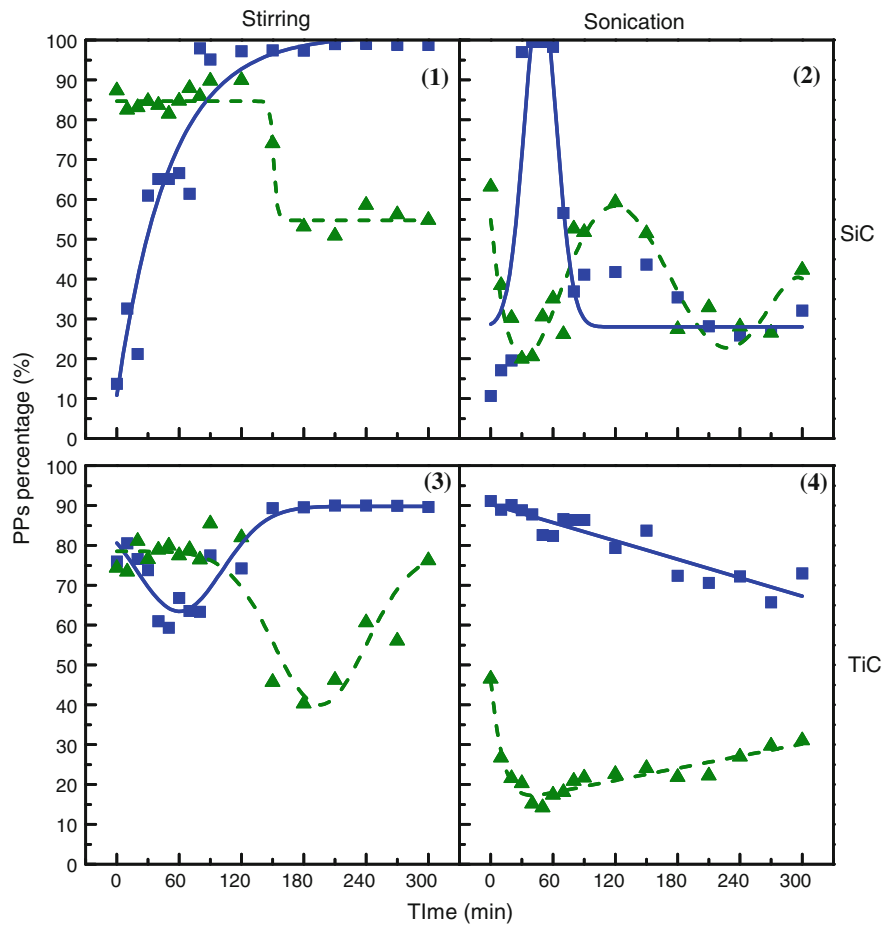

opposition to strong variation with time for the $\mathrm{SiC}$ NPs case.

\section{Surface composition after pre-dispersion}

The surface composition was measured to evaluate the changes produced by the protocol of dispersion on the surface of $\mathrm{SiC}$ and TiC NPs (PPs and agglomerates) dispersed in water versus those obtained in pluronic dispersions.

Figure 4 shows the evolution of the surface composition of $\mathrm{Si} / \mathrm{C}$ and $\mathrm{Ti} / \mathrm{C}$ ratio computed from the XPS determination (in at.\%) of the surface concentration as indicated in Table 1.

No clear difference between $\mathrm{SiC}$ and $\mathrm{TiC}$ chemical composition with time is observed in water. $\mathrm{Si} / \mathrm{C}$ ratio is close to the pristine material both for stirring and sonication methods. TiC surface composition is the one of pristine material in water, but a slight decrease of the $\mathrm{C}$ concentration (higher $\mathrm{Ti} / \mathrm{C}$ ratio) is observed for sonication. XPS also shows that the surface is composed of $\mathrm{TiO}$ and $\mathrm{TiO}_{2}$.

A remarkably different behavior is observed for the dispersion in pluronic: $\mathrm{SiC}$ and $\mathrm{TiC}$ behave differently timewise. On the one hand, Ti/C time-ratio is either stable (stirring) or slightly increases $(10 \%$ increase over $300 \mathrm{~min}$ for sonication). For both cases, we observe a reduction of $\mathrm{C}$ compared to pristine material. On the other hand, substantial enrichment in carbon of the SiC NPs surface for both stirring and sonication takes place with time dependence in the case of stirring. XPS also shows that the surface is composed of $\mathrm{SiC}$ and a mixture of $\mathrm{SiO}_{2} \mathrm{C}_{2}+\mathrm{SiOC}_{3}$, commonly presented as $\mathrm{SiC}_{x} \mathrm{O}_{y}$ (Lee et al. 2009; Shimoda et al. 2007).

We also measured the metal release by AAS in the various dispersants by stirring and sonication (see Supplemental data). TiC does not show any ion release within the limit of detection (few ppm). SiC is not stable compared to TiC: in water, we measured $\mathrm{Si}$ ion release whose concentration decreases with time. This indicates some cleaning effects, which were greater for sonication compared to stirring. In pluronic, no ion release was detected.

\section{During the in vitro assays}

As previously described, methods of dispersion (sonication, stirring, presence of dispersant, etc.) can affect the PSD. In order to evaluate whether these modifications could have an impact on the potential toxic and pro-inflammatory effects of $\mathrm{SiC}$ and $\mathrm{TiC}$ NPs on 
Fig. $4 \mathrm{Si} / \mathrm{C}$ and $\mathrm{Ti} / \mathrm{C}$ ratios from surface composition in water (closed square) and pluronic (closed triangle) dispersions, obtained from XPS measurements. SiC NPs: stirring (1) and sonication (2). Line A corresponds to the pristine $\mathrm{Si} / \mathrm{C}$ ratio. TiC NPs: stirring (3) and sonication (4). Line $B$ corresponds to the pristine $\mathrm{Ti} / \mathrm{C}$ ratio. Trend lines are intended to guide the eyes of the reader

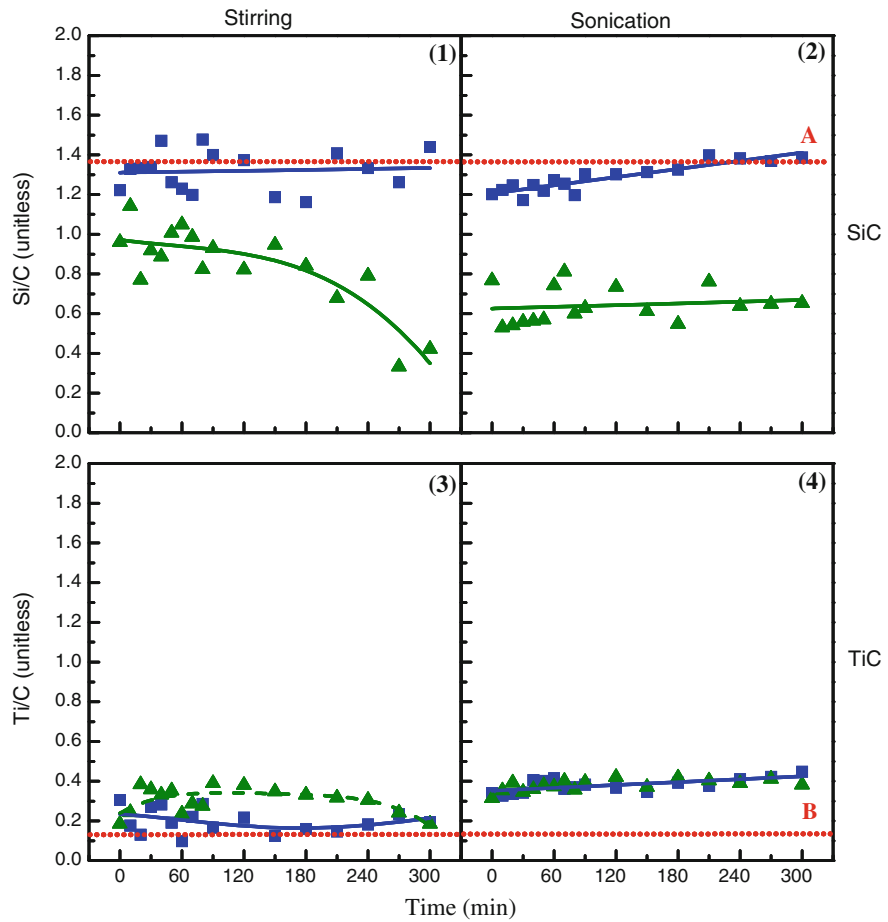

human immortalized keratinocytes, the different $\mathrm{SiC}$ and $\mathrm{TiC}$ NPs dispersions were diluted at $100 \mu \mathrm{g} / \mathrm{mL}$ in culture media. For practical reasons, the pre-dispersions were prepared using a time of $30 \mathrm{~min}$. Keratinocytes were incubated in the presence of $\mathrm{TiC}$ or $\mathrm{SiC}$ NPs for $6,24,40$, or $48 \mathrm{~h}$. First, we collected two different portions (CMP and cells portion as described in the section "Sample preparation for characterization") and evaluated the PSD of NPs in each portion.

Figure 5 shows the hydrodynamic size of the PPs of both defined portions.

Once again, a different behavior is observed when comparing $\mathrm{SiC}$ and $\mathrm{TiC}$. On the one hand, $\mathrm{SiC}$ dispersion in culture medium induces an immediate increase of the average PPs diameter by about $40 \%$ (from 25 to $35 \mathrm{~nm}$ ), whatever the pre-dispersion dispersant or method. Long dispersion time induces a variation of up to $15 \%$ of the average size. On the other hand, TiC dispersion in culture medium induced a slight variation $(10 \%)$ around the averaged NPs hydrodynamical diameter.

Figure 6 shows the percentage of PPs (from number related distributions) in each defined portion.

Generally speaking, the longer the incubation time, the lower the PPs ratio in medium and the higher the ratio of NPs exposed to the cells. For TiC, the same time pattern is observed whatever the conditions: it first increases to reach a "plateau" after $36 \mathrm{~h}$, at which time cell exposure is stable, reaching up to $90 \%$ of the dispersed PPs (except for sonication in pluronic which is slightly lower).

For $\mathrm{SiC}$, the time pattern shows an oscillating behavior observed whatever the pre-dispersion method, indicating that the dose given to the cell is not constant and is a fraction of the dispersed amount. One has to wait at least $48 \mathrm{~h}$ to make sure that cells are exposed to $80 \%$ of the PP. The sonication probe seems to speed up the cell exposition to NP compared to stirring.

Immediate comparison of the surface composition to pristine material shows that the metal/C ratio is reduced, on average, by a factor of 45 for $\mathrm{SiC}$ and by 85 for $\mathrm{TiC}$ (data not shown). This can only be associated with a large increase of $\mathrm{C}$ concentration masking effect, which in turn can only originate from a wrapping of the NP by a coating from the culture medium (which indicates the presence of $\mathrm{N}, \mathrm{P}, \mathrm{Ca}, \mathrm{S}$, and some $\mathrm{Cl}$ ). Within the XPS sensitivity, it is also seen that the masking effect is time dependent. Nevertheless, due to the very low amount of the metal as measured on the XPS surveys, we will further assume that it is completely masked by the culture-medium coating. 


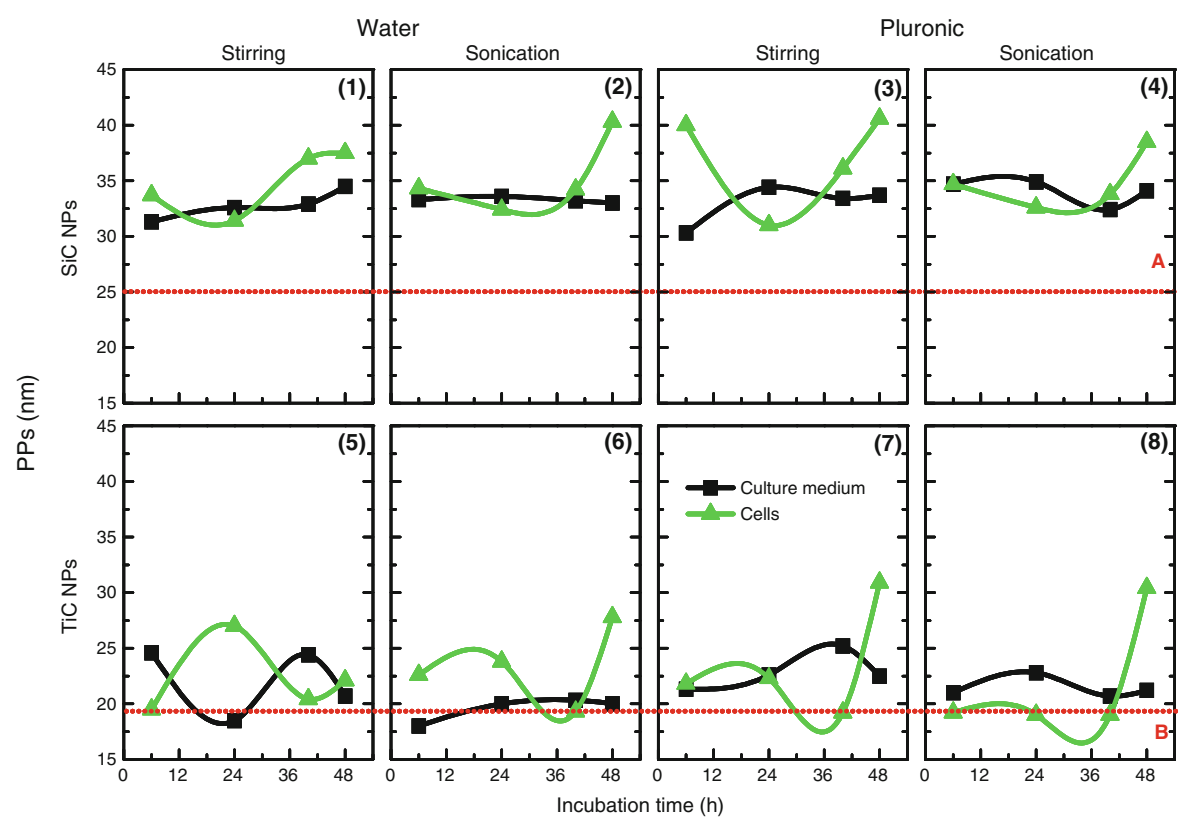

Fig. 5 Hydrodynamic size of NPs obtained from CLS measurements during in vitro assays. SiC NPs: stirring in water (1), sonication in water (2), stirring in pluronic (3), sonication in pluronic (4). Line A corresponds to the SiC PPs pristine value.
TiC NPs: stirring in water (5), sonication in water (6), stirring in pluronic (7), sonication in pluronic (8). Line B corresponds to the TiC PPs pristine value. Trend lines are intended to guide the eyes of the reader

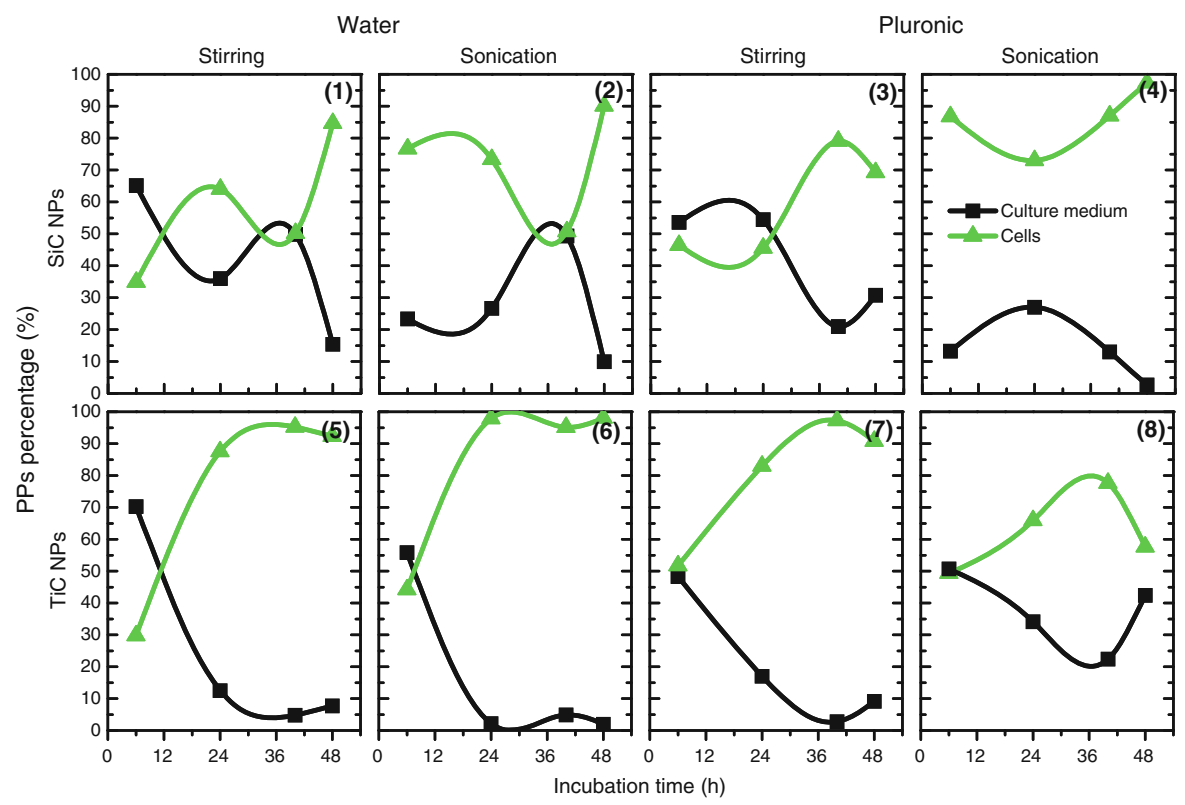

Fig. 6 Percentage of PPs in the CMP and the CP, from PSD obtained from CLS measurements. SiC NPs: stirring in water (1), sonication in water (2), stirring in pluronic (3), sonication in pluronic (4). TiC NPs: stirring in water (5), sonication in water (6), stirring in pluronic (7), sonication in pluronic (8). Trend lines are intended to guide the eyes of the reader 
Cell exposure

For the in vitro cell exposure assay, NPs were predispersed for $30 \mathrm{~min}$ in water or pluronic by the two methods and incubated for $48 \mathrm{~h}$.

The cytotoxic and pro-inflammatory effects of the different NP dispersions on human keratinocytes were then assayed by measuring the LDH and IL-8 releases, respectively (Fig. 7). As shown in Fig. 7(1), both $\mathrm{SiC}$ and TiC NPs induced a slight increase in the amount of LDH released (independent of the dispersion method) in comparison to the highly toxic positive control BC (Vankoningsloo et al. 2010). Firstly, SiC NPs dispersed in the presence of pluronic induced an increased release of $\mathrm{LDH}$ in comparison with those dispersed in water at each time of incubation. Presence of pluronic decreases the amount of LDH released after incubation with TiC NPs which are only stirred (method 1), while an increase is observed after sonication (method 2). Secondly, sonication slightly decreases the cytotoxic effect of SiC NPs (in the presence or not of pluronic) after $24 \mathrm{~h}$ of incubation, which is no longer observed at greater times. Cytotoxic effects are milder after sonication for waterdispersed TiC NPs (at each time of incubation), while sonication does not alter on average the toxic effect of pluronic-dispersed TiC NPs. Similar results were obtained in IL-8 measurements for both NPs (Fig. 7(2)).

\section{Discussion}

For the sake of discussion, we will summarize our data in a graphical view to derivate general trends. We will do that by presenting "time evolution patterns" of variables such as PPs diameter, PPs percentage, and $\mathrm{me} / \mathrm{C}$ ratio, as well as a "qualitative" evolution of those variables over $300 \mathrm{~min}$ for pre-dispersion, 24 and $48 \mathrm{~h}$ of incubation. This is presented in Table 2. One can read the table as follows, for instance: TiC NPs, pre-dispersed in water by stirring, during in vitro incubation evolves from lower to higher PPs percentages.

General observation is that $\mathrm{SiC}$ and $\mathrm{TiC}$ behavior is time dependent, and the time pattern is different from $\mathrm{SiC}$ to $\mathrm{TiC}$.

One of the main features of our results is that surface composition evolves with the time and the pre- dispersion technique. While stirring in water-based dispersions have basically no effect, the use of pluronic or sonication drastically modifies the $\mathrm{SiC}$ or TiC surface concentration. This is confirmed by HRTEM experiments on selected samples of $\mathrm{SiC}$ and TiC NPs pre-dispersed in water and pluronic (see Fig. 8).

Figure 8(1) shows a dispersion of SiC NPs in water by stirring, where a smooth and even surface is observed, while for the pluronic-based dispersion NPs are covered with an amorphous layer (see Fig. 8(3)). Sonication in water dispersion seems to produce a degradation/erosion in the surface of the NPs (see Fig. 8(2)), because of the high-energy provided (Raman and Abbas 2008), and in pluronic it produces an adsorbed amorphous outer layer (see Fig. 8(4)). The outer layer thickness can be estimated at around $5 \mathrm{~nm}$, and the XPS analysis indicates that this adsorbed layer is formed of $\mathrm{C}$ and $\mathrm{O}$, principal components of the Pluronic F108.

TiC NP dispersions obtained by stirring and sonication were also compared by HRTEM. Stirring in water seems to have produced a cleaning (Fig. 8(5)), while an irregular surface is observed when pluronic was used (Fig. 8(7)). Sonication produces an irregular surface, both in water (Fig. 8(6)) and pluronic (Fig. 8(8)); interestingly, more adsorption is observed for the stirring condition.

This cleaning/adsorption effect explains the variations of metal/C ratio depicted in Table 2 and measured by XPS. Indeed, when high-energy dispersion methods are used, erosion seems to remove more $\mathrm{C}$ from the surface and the core of pristine NPs than the readily adsorbed $\mathrm{C}$ from the Pluronic F108. The data for sonication in pluronic show that the adsorption process may form multiple layers of Pluronic F108 molecules, which could be attached or detached depending on the duration of the sonication.

According to the formation enthalpies for both components, $\mathrm{SiC}$ NPs (pure $\mathrm{SiC}-73.18 \mathrm{~kJ} / \mathrm{mol}$ ) are less stable than TiC NPs (pure TiC $-142.3 \mathrm{~kJ} / \mathrm{mol}$ ), which corroborates the degradation of $\mathrm{SiC}$ surface. If $\mathrm{O}$, was only in the chemical form of $\mathrm{SiO}_{2}$, it would be very stable $(\Delta H=-838.0 \mathrm{~kJ} / \mathrm{mol})$. However, XPS data show that $\mathrm{O}$ is also chemically bounded to both $\mathrm{C}$ and $\mathrm{Si}$ to form $\mathrm{Si}_{x} \mathrm{C}_{y} \mathrm{O}_{z}$ that is thermodynamically unstable $(+161 \mathrm{~kJ} / \mathrm{mol})$, confirming that the outer surface is prone to dissolution. This is once again illustrated with the measurements of the zeta 

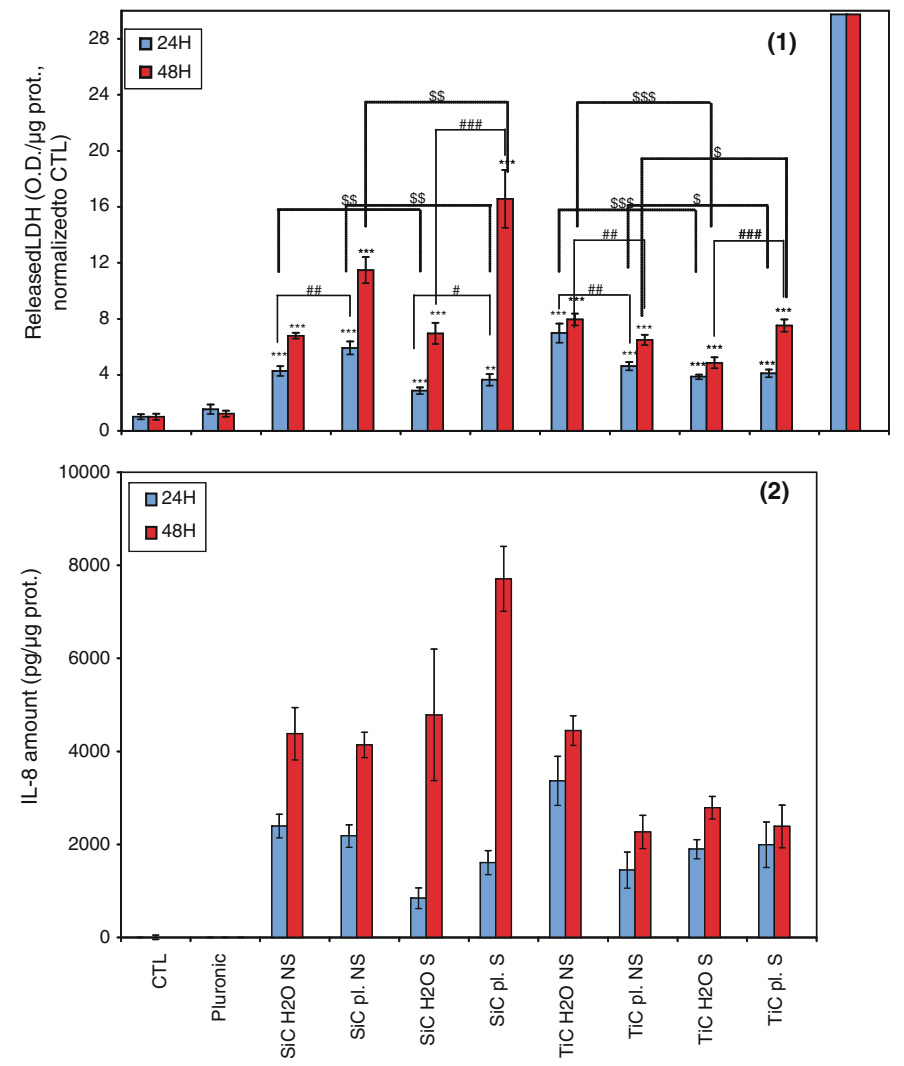

Fig. 7 Effect of $\mathrm{SiC}$ and TiC NPs on LDH release (1) and on IL8 protein release (2) from N-hTERT keratinocytes. N-hTERT keratinocytes were incubated for $24 \mathrm{~h}$ in the presence or absence (CTL) of different suspensions of SiC or TiC NPs at $100 \mu \mathrm{g} / \mathrm{mL}$. Cytotoxicity was evaluated by measuring the amount of LDH released in culture medium (1). Results in optical density (OD) units $/ \mu \mathrm{g}$ proteins were expressed as means $\pm \operatorname{SD}$ for $n=4$, and presented as percentages of controls. ${ }^{* *} p<0.01$ versus corresponding CTL; $* * * p<0.001$ versus corresponding CTL.

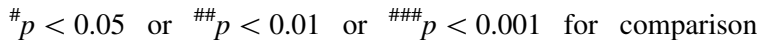
between water and pluronic dispersions. $\$ p<0.05$ or $\$ \$ p<0.01$ or $\$ \$ p<0.001$ for comparison between stirring and four (BC, $10 \mu \mathrm{g} / \mathrm{mL}$ ) values: $24 \mathrm{~h}: 39.87 \pm 3.8$; $48 \mathrm{~h}$ : $79.91 \pm 10.48$. Pro-inflammatory effects of different suspensions of $\mathrm{SiC}$ or TiC NPs were investigated by measuring the amount of IL-8 released in culture medium (2). Results in pg IL$8 / \mathrm{mg}$ proteins were expressed as means \pm SD for $n=4$

Table 2 Time pattern evolution of the NPs in pre-dispersion ${ }^{\mathrm{a}}$ and during in vitro incubation ${ }^{\mathrm{b}}$

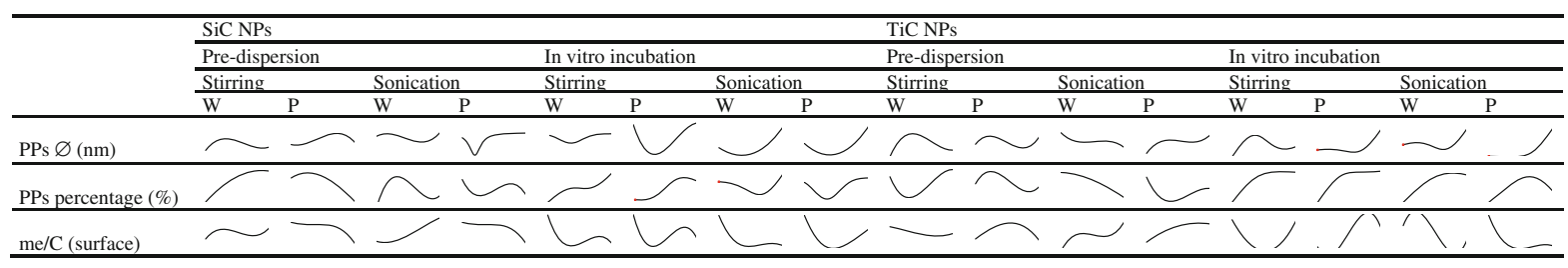

$W$ water, $P$ Pluronic F108

a Over $300 \mathrm{~min}$

b Over $48 \mathrm{~h}$

potentials of $\mathrm{SiC}$ and $\mathrm{TiC}$ pluronic dispersion at $60 \mathrm{~min}$ of application of stirring or sonication. It gives values of -14.61 and $0.81 \mathrm{mV}$, respectively, for $\mathrm{SiC}$.
In the case of dispersed TiC NPs, the zeta potential values obtained for the same conditions are -17.74 and $-13.32 \mathrm{mV}$, respectively. These values are 
Fig. 8 HRTEM images. SiC NPs: stirring in water (1), sonication in water (2), stirring in pluronic (3), sonication in pluronic (4). TiC NPs: stirring in water (5), sonication in water (6), stirring in pluronic (7), sonication in pluronic (8). Scale bars correspond to $10 \mathrm{~nm}$
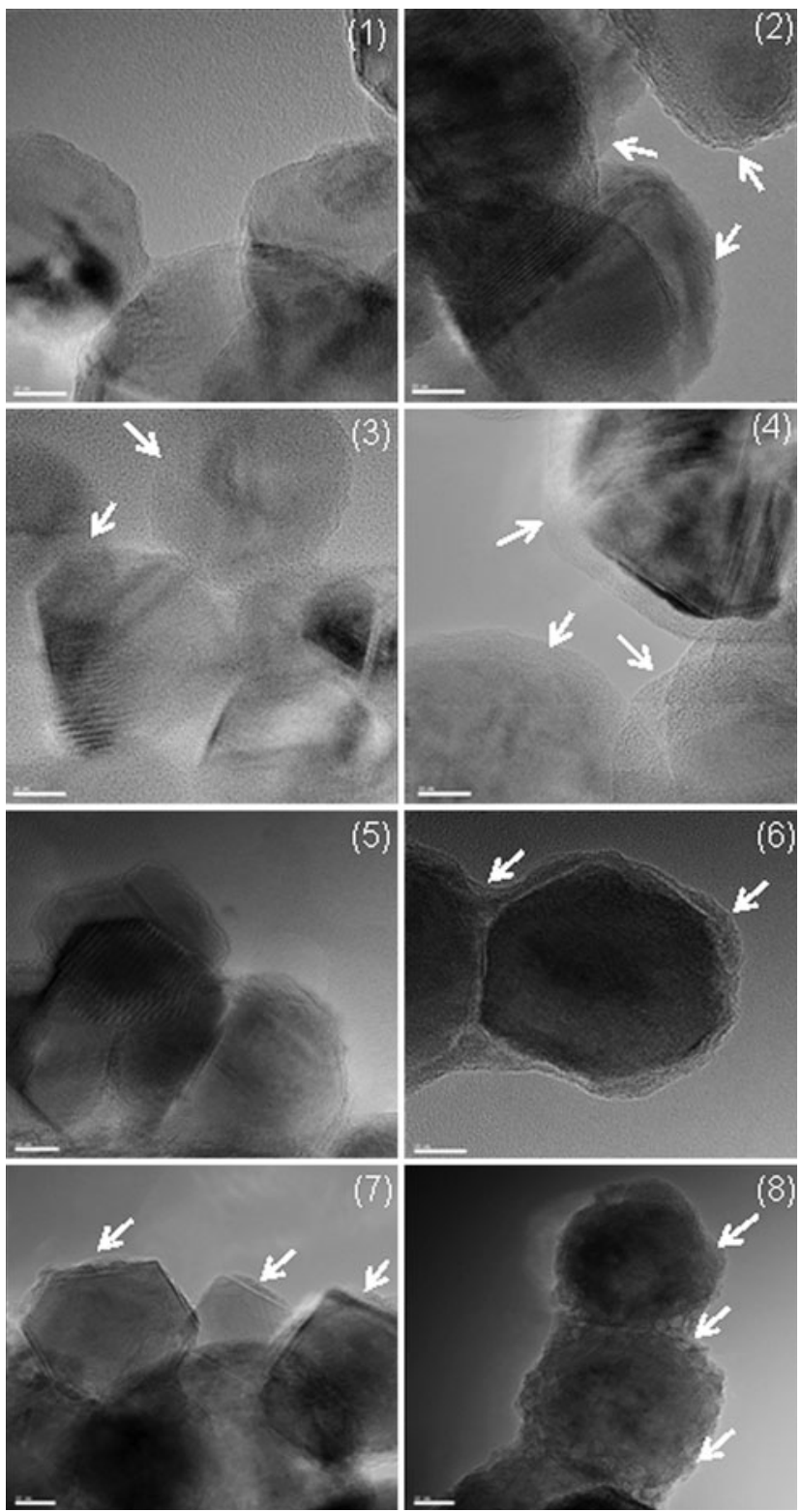

similar and illustrate that TiC NPs are less affected for the protocol of dispersion.

If we observe the PPs percentage over $300 \mathrm{~min}$ for the pre-dispersed material, it is seen that pluronic reduces systematically the concentration of PPs (one has to compare the initial and final concentration in Table 2). This agglomeration phenomenon promoted by the presence of Pluronic F108 molecules indicates that Pluronic F108 is not an adequate dispersant for carbide NPs. On the contrary, water predispersions produce higher PPs percentages. TiC shows the same time pattern whatever the dispersion technique, but with the final PPs percentage more in relation with the medium than the energy provided to the solution. For this reason, we believe that PPs percentage of surface-stable NPs (or dose given to the cells by surface-stable NPs) is at first driven by the medium. For the unstable surface-NPs, SiC, the PPs percentage is at first driven by the dispersion method (or the energy provided) that induces complex chemical changes of the surface through successive mechanism of dissolution and wrapping of both the NPs and the agglomerates (see info about agglomerates in Fig. 2). 
The fate of the already presented PPs or generated PPs is resumed at Fig. 9. For $\mathrm{SiC}$ in water dispersions, NP surface is cleaned (removal of $\mathrm{Si}, \mathrm{SiO}, \mathrm{SiO}_{2}$, and $\mathrm{Si}_{x} \mathrm{C}_{y} \mathrm{O}_{z}$ traces) by stirring, and some erosion is produced under sonication. Pluronic- and stirring based-dispersions produces adsorption of Pluronic F108 molecules and sonication produces adsorption/ erosion. An additional evidence of the method driven modifications is the release of $\mathrm{Si}^{4+}$ ions in water predispersion as demonstrated by AAS results. The ion release is method dependent and decrease with time (see Supplementary Fig. 4). This can be explained as the sonication produce cavitation/erosion that promotes the removal of $\mathrm{Si}$ and $\mathrm{SiO}_{2}$ traces, as well as the $\mathrm{SiC}_{x} \mathrm{O}_{y}$ layer from the surface (Suslick et al. 1999; Suslick and Price 1999). Then, the core of the NPs is reached, where more $\mathrm{C}$ (lower $\mathrm{Si} / \mathrm{C}$ ratio) is present (Tartaj et al. 1998); thus, less $\mathrm{Si}$ is available for dissolution as ions.

For $\mathrm{TiC}$, water pre-dispersion cleans the NP surfaces whatever the method. In the case of pluronic dispersion, adsorption of pluronic molecules is finally observed as a nearly wrapping coating for stirring, and as local molecule attachments for sonication. These results are in agreement with previous reports, evidencing that surface modifications are produced when $\mathrm{SiC}$ and TiC NPs are dispersed in aqueous suspensions prior to in vitro assays (Novak et al. 2007).

As explained in the previous sections, in vitro bioassays have been performed for a 30-min predispersed material and 24-48 $\mathrm{h}$ incubation time with N-hTERT cells. Table 3 resumes the evolution of the properties of the material used to perform the bioassays on these chosen conditions.

If one look at the surface composition first, one can see that after stirred water pre-dispersion and incubation, metal is still detected at the surface. On the contrary, it is not present anymore when pre-dispersion occurred in stirred pluronic. This indicates that culture-medium components absorb in a more pronounced way on the pluronic already pre-coated NPs than on the carbides. On the contrary, sonication predispersion in water or pluronic renders the surface less prone for the so-called "protein corona" absorption (Lundqvist et al. 2008) given that metal is still present at the surface of the NPs, even after $24 \mathrm{~h}$ of incubation in culture media. This suggests a not fully covering protein wrapping. The protein corona induces an increase of the hydrodynamic diameter of about
$10 \mathrm{~nm}$ in all cases. This has already been observed by Monopoli et al. (2011) for silica NPs.

In addition, from the zeta potential variations measured, we are likely breaking the molecular chains of the pluronic remaining in dispersion or adsorbed into the NPs surface. We hypothesize that the strong energy input of the sonication is the cause. Variations in the $\mathrm{pH}$ of the pre-dispersions were also measured, i.e., the $\mathrm{pH}$ of pluronic pre-dispersion varies from 6.6 to 5.1 units after $90 \mathrm{~min}$ of sonication. This is in line with similar variations reported, where the zeta potential, the $\mathrm{pH}$, and the concentration of the dispersion were accounting for modifications on the NP surface (Che et al. 2007; Singh et al. 2007). Indeed, the significant effects produced by the use of sonication, i.e., degradation/erosion of the surface of the $\mathrm{SiC}$ and TiC NPs and adsorption of Pluronic F108 molecules, should serve as a warning to use this methods with caution, no matter the time of application used. In a previous communication, we reported analogous conditions for multiwall carbon nanotubes in similar dispersions (Mejia et al. 2011).

The results of the screening of the effects produced on the PSD and the surface composition of NPs by two commonly used protocols of pre-dispersion in water and pluronic dispersions still reveals that the chemical complexity of the prepared dispersions, whatever the method used and given the modifications found, should be reduced as much as possible (Taurozzi et al. 2011).

PPs percentage measurements (see Table 3) clearly demonstrate that the dose delivered to the cells is NPs type dependant: cells exposition to $\mathrm{TiC}$ is always larger than $\mathrm{SiC}$ even for the same protocol of dispersion. The main parameter that drives the $\mathrm{SiC}$ PPs percentage in the CMP seems to be the energy delivered during the pre-dispersion phase. Indeed, stirring always produces lower PPs percentages than sonication whatever the pre-dispersion medium. On the contrary, the main parameter that seems to drive the TiC PPs percentage is the pre-dispersion medium: pluronic always produce a lower PPs percentage than its water counterpart, whatever the stirring method.

This data suggest that the dose available for the cells need to be adequately measured or at least estimated cautiously. Some efforts were already produced in the literature (Lison et al. 2008; Teeguarden et al. 2007), yet the behavior assessment of several NP dispersions is still necessary. 
Fig. 9 Proposed model of the effects observed, based on the PSD and the surface composition evaluation, in NP dispersions prepared with different protocols of dispersion. SiC NP dispersion (1), TiC NP dispersions (2)

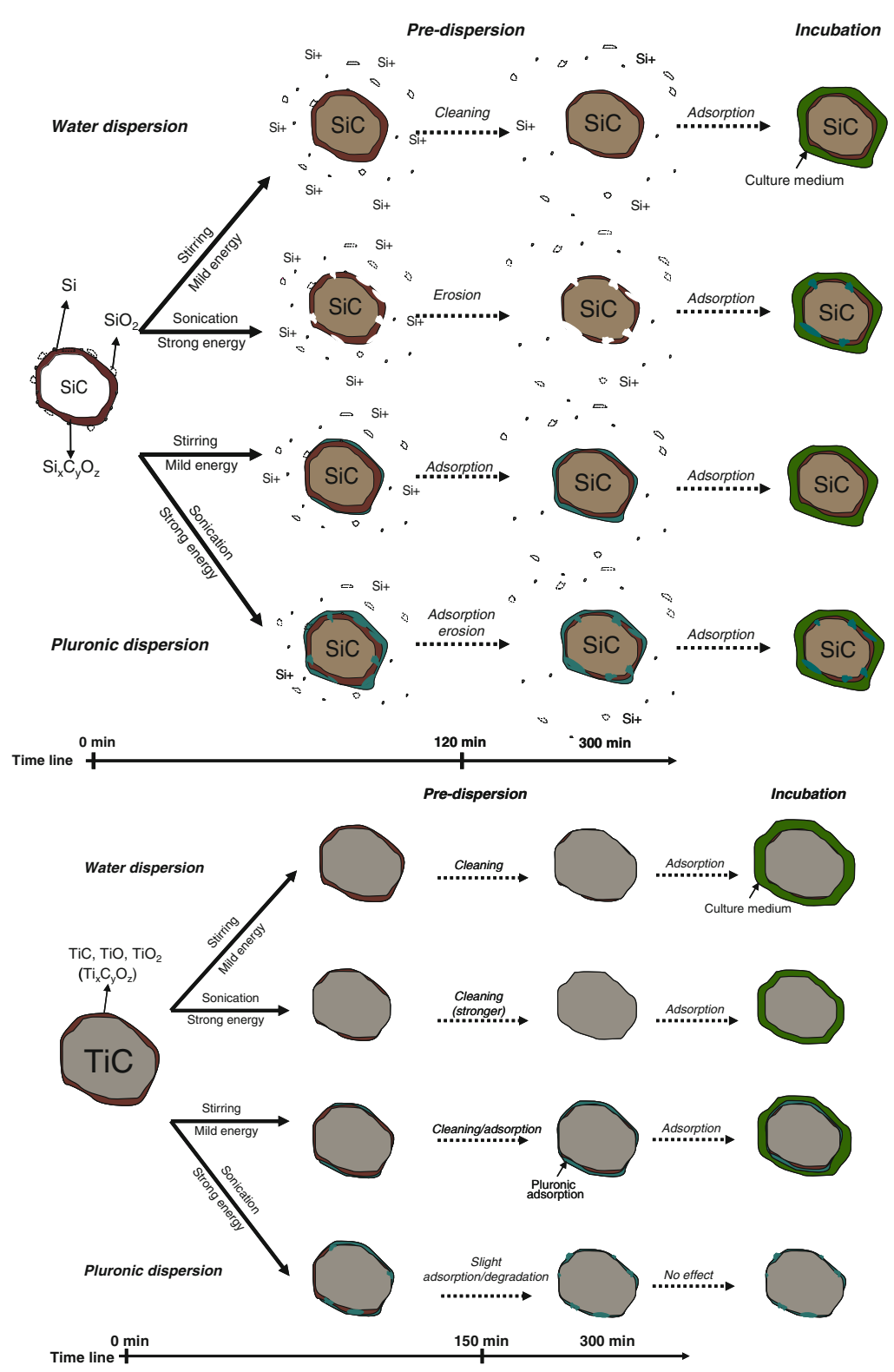

The $\mathrm{SiC}$ or TiC NPs that was finally in contact with cells during the bioassay evaluation differs only by surface chemical composition and PPs percentage. Indeed, the average hydrodynamic size is about the same for all pre-dispersion conditions (see Table 2).

First observation of IL8 and LDH release (Fig. 7) is that even if a nano-material is wrapped by a "biocompatible dispersant," it may induce some inflammation and reduction in viability (see, for example, the results of $\mathrm{SiC}$ or $\mathrm{TiC}$ sonicated in pluronic), while the dispersant has no effect by itself.
Several reasons may account for that: the surface chemical composition of the wrapped material is not the one of the dispersant; the shape has a strong effect or the dose delivered is not the one that was accounted for.

For example, our results show that SiC NPs predispersed in the presence of pluronic by stirring induced higher LDH release than those dispersed in water. Because the final size are the same, the PPs percentage of $\mathrm{SiC}$ in pluronic wrapped is lower than the one pre-dispersed in water $(40 \%$ for pluronic, 


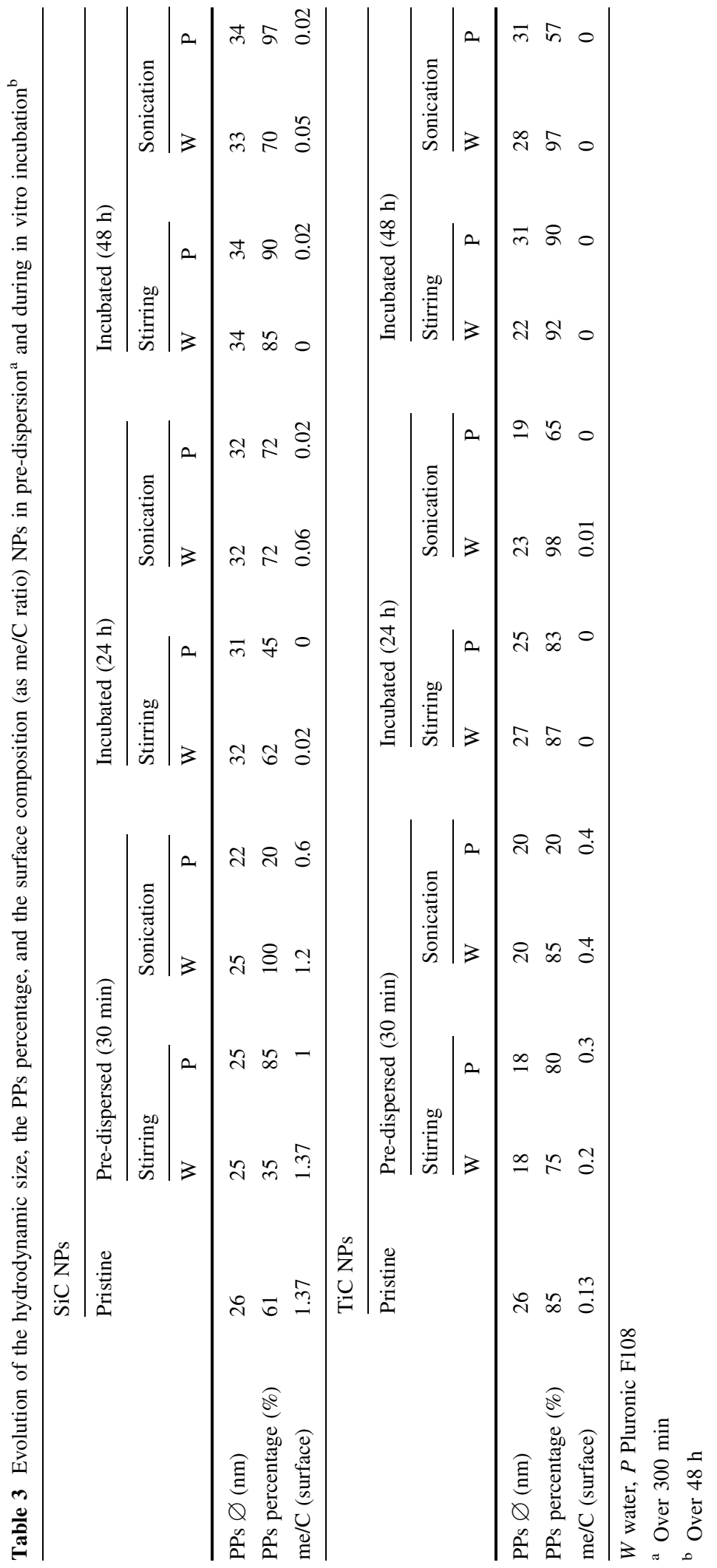


$65 \%$ for water), one can deduce that only less $\mathrm{Si}$ in surface is the driving parameter to account for the higher LDH and IL-8 release. Thus, the toxicity effect seems to come from the pluronic adsorbed on the NP surface which generates the $\mathrm{Si}_{x} \mathrm{C}_{y} \mathrm{O}_{z}$ compounds (see Fig. 4(8)). Surprisingly, the contrary is observed for TiC NPs. In the case of water-based dispersions, where no bias is added, the differences observed in the LDH and IL-8 release are less significant; yet, they can be attributed only to the protocol of dispersion.

Accordingly, the use of Pluronic F108 or other surfactants, as well as the use of invasive methods of dispersions, should be avoided in order to only test the effect of the NPs. In addition, Meißner et al. (2010) have clearly stated that proteins may influence the cellular uptake and the biological activity of nanomaterials. Nevertheless, information from PSD and surface composition modifications produced before the actual in vitro assays is still lacking. The information provided in the present report constitutes a starting point to study the role of protein and/or adsorbed species (Fubini et al. 2010; Lundqvist et al. 2008), using the PSD and the surface parameters as key parameters.

\section{Conclusions}

Nowadays, to perform in vitro nanotoxicology assays, a biocompatible medium, a dispersion technique, and a time of dispersion have to be chosen. A reasonable question then occurs: will the NP physicochemical properties be unaffected by such a selection.

In this study, we demonstrated that the integrity of nanomaterials may differ strongly when dispersed in water or Pluronic F108 solution by either stirring or sonication methods. We investigated two commercial carbides ( $\mathrm{SiC}$ and $\mathrm{TiC} \mathrm{NPs}$ ) and found that the evolution of their physicochemical properties (size, percentage of PPs, surface composition, etc.) over 300 min time span is medium driven for $\mathrm{SiC}$ and "method of dispersion" driven for TiC.

At first, this is related to the chemical stability of the nanomaterial extreme surface: a chemically stable outer shell will be mostly affected by the power transferred during the dispersion method, while an unstable one will be mostly affected by the chemical compounds present in the dispersion used. At second, concurrent effects like cleaning and wrapping are also observed depending on the selection of the dispersion medium (e.g., water or pluronic).

Finally, the NP physicochemical properties are further on altered when in vitro incubation for toxicity assessment is performed. This has an impact on the amount (dose) of NPs in dispersion and in contact with cells affecting strongly the biological response as highlighted by the IL- 8 and LDH release tests.

A qualitative model describing the modifications the NPs undergo during pre-dispersion and in vitro assays was presented. This study highlights the need to evaluate the toxicity of nanomaterials on a case-bycase study even within the framework of a standardized protocol and material family.

Acknowledgments This work is supported by the "Direction Générale des Technologies de la Recherche et de l'Energie" (DGTRE) of the Walloon Region of Belgium (Nanotoxico Project, RW/FUNDP research convention No. 516252). O. Toussaint is a Research Associate of the Belgian FRS/ FNRS. The authors acknowledge financial support from the European Union under the Framework 6 program under a contract for the Integrated Infrastructure Initiative. Reference 026019 ESTEEM and Framework 7 program, Qnano (INFRA STRUCTURE-2010-1-262163).

\section{References}

Bae S-T, Shin H, Jung HS, Hong KS (2009) Synthesis of titanium carbide nanoparticles with a high specific surface area from $\mathrm{TiO}_{2}$ core-sucrose shell precursor. J Am Ceram Soc 92(11):2512-2516

Barillet S, Jugan ML, Laye M, Leconte Y, Herlin-Boime N, Reynaud C, Carrière M (2010) In vitro evaluation of SiC nanoparticles impact on A549 pulmonary cells: Cyto-, genotoxicity and oxidative stress. Toxicol Lett 198(3): 324-330

Bihari P, Vippola M, Schultes S, Praetner M, Khandoga A, Reichel C, Coester C, Tuomi T, Rehberg M, Krombach F (2008) Optimized dispersion of nanoparticles for biological in vitro and in vivo studies. Part Fibre Toxicol 5(1):14

Borm P, Robbins D, Haubold S, Kuhlbusch T, Fissan H, Donaldson K, Schins R, Stone V, Kreyling W, Lademann J, Krutmann J, Warheit D, Oberdorster E (2006) The potential risks of nanomaterials: a review carried out for ECETOC. Part Fibre Toxicol 3(1):11

Che J, Wang X, Xiao Y, Wu X, Zhou L, Yuan W (2007) Effect of inorganic-organic composite coating on the dispersion of silicon carbide nanoparticles in non-aqueous medium. Nanotechnology 18(13):135706

Cherukuri P, Bachilo SM, Litovsky SH, Weisman RB (2004) Near-infrared fluorescence microscopy of single-walled carbon nanotubes in phagocytic cells. J Am Chem Soc 126(48):15638-15639 
Cherukuri P, Gannon CJ, Leeuw TK, Schmidt HK, Smalley RE, Curley SA, Weisman RB (2006) Mammalian pharmacokinetics of carbon nanotubes using intrinsic near-infrared fluorescence. Proc Natl Acad Sci USA 103(50): 18882-18886. doi:10.1073/pnas.0609265103

Corey JM, Gertz CC, Sutton TJ, Chen Q, Mycek KB, Wang BS, Martin AA, Johnson SL, Feldman EL (2009) Patterning N-type and S-type neuroblastoma cells with Pluronic F108 and ECM proteins. J Biomed Mater Res A 93(2):673-686

Dickson MA, Hahn WC, Ino Y, Ronfard V, Wu JY, Weinberg RA, Louis DN, Li FP, Rheinwald JG (2000) Human keratinocytes that express hTERT and also bypass a p16INK4a-enforced mechanism that limits life span become immortal yet retain normal growth and differentiation characteristics. Mol Cell Biol 20(4):1436-1447. doi:10.1128/mcb.20.4.1436-1447.2000

Foucaud L, Wilson MR, Brown DM, Stone V (2007) Measurement of reactive species production by nanoparticles prepared in biologically relevant media. Toxicol Lett 174: $1-9$

Fubini B, Ghiazza M, Fenoglio I (2010) Physico-chemical features of engineered nanoparticles relevant to their toxicity. Nanotoxicology 4(4):347-363

Fukushima M, Zhou Y, Yoshizawa Y-I (2008) Fabrication and microstructural characterization of porous silicon carbide with nano-sized powders. Mater Sci Eng B 148(1-3):211-214

Guevara-Lora I, Czosnek C, Smycz A, Janik J, Kozik A (2009) $\mathrm{SiC}$ nanoparticles as potential carriers for biologically active substances. J Phys Conf Ser 146(1):012022

Hassan M, Rawat RS, Lee P, Hassan SM, Qayyum A, Ahmad R, Murtaza G, Zakaullah M (2008) Synthesis of nanocrystalline multiphase titanium oxycarbide $\left(\mathrm{TiC}_{x} \mathrm{O}_{y}\right)$ thin films by UNU/ICTP and NX2 plasma focus devices. Appl Phys A 90(4):669-677

Hoet P, Bruske-Hohlfeld I, Salata O (2004) Nanoparticlesknown and unknown health risks. J Nanobiotechnol 2(1): 12

Iijima M, Kamiya H (2008) Surface modification of silicon carbide nanoparticles by azo radical initiators. J Phys Chem C 112(31):11786-11790

Iolitec (2007a) Silicon(IV)carbide-technical data sheet. http://www.nanomaterials.iolitec.de/en/Nanomaterials/ carbide.html. Retrieved 2012-03-19

Iolitec (2007b) Titanium(IV)carbide powder-technical data sheet. http://www.nanomaterials.iolitec.de/en/Nanomater ials/carbide.html. Retrieved 2012-03-19

Karakoti A, Hench L, Seal S (2006) The potential toxicity of nanomaterials - the role of surfaces. J Manag 58(7):77-82

Lee K-H, Lee S-K, Jeon K-S (2009) Photoluminescent properties of silicon carbide and porous silicon carbide after annealing. Appl Surf Sci 255(8):4414-4420

Lin H, Gerbec JA, Sushchikh M, McFarland EW (2008) Synthesis of amorphous silicon carbide nanoparticles in a low temperature low pressure plasma reactor. Nanotechnology 19(32):325601

Lison D, Thomassen LCJ, Rabolli V, Gonzalez L, Napierska D, Seo JW, Kirsch-Volders M, Hoet P, Kirschhock CEA, Martens JA (2008) Nominal and effective dosimetry of silica nanoparticles in cytotoxicity assays. Toxicol Sci 104(1):155-162. doi:10.1093/toxsci/kfn072
Lundqvist M, Stigler J, Elia G, Lynch I, Cedervall T, Dawson KA (2008) Nanoparticle size and surface properties determine the protein corona with possible implications for biological impacts. Proc Natl Acad Sci 105(38):1426514270. doi:10.1073/pnas.0805135105

Meißner T, Kühnel D, Busch W, Oswald S, Richter V, Michaelis A, Schirmer K, Potthoff A (2010) Physical-chemical characterization of tungsten carbide nanoparticles as a basis for toxicological investigations. Nanotoxicology 4(2):196-206. doi:10.3109/17435391003605455

Mejia J, Tichelaar F, Saout C, Toussaint O, Bernard M, Mekhalif Z, Lucas S, Delhalle J (2011) Effects of the dispersion methods in Pluronic F108 on the size and the surface composition of MWCNTs and their implications in toxicology assessment. J Nanopart Res 13:655-667. doi: 10.1007/s11051-010-0063-7

Merkus HG (2009) Particle size measurements—fundamentals, practice, quality. Particle technology series, vol 17. Springer, Dordrecht, pp 329-334. doi:978-1-4020-9016-5

Monopoli MP, Walczyk D, Campbell A, Elia G, Lynch I, Baldelli Bombelli F, Dawson KA (2011) Physical-chemical aspects of protein corona: relevance to in vitro and in vivo biological impacts of nanoparticles. J Am Chem Soc 133(8):2525-2534

Nel A, Xia T, Madler L, Li N (2006) Toxic potential of materials at the nanolevel. Science 311(5761):622-627. doi: 10.1126/science. 1114397

Novak S, Kovac J, Drazic G, Ferreira JMF, Quaresma S (2007) Surface characterisation and modification of submicron and nanosized silicon carbide powders. J Eur Ceram Soc 27(12):3545-3550

Piret JP, Detriche S, Vigneron R, Vankoningsloo S, Rolin S, Mejia Mendoza J, Masereel B, Lucas S, Delhalle J, Luizi F, Saout C, Toussaint O (2010) Dispersion of multi-walled carbon nanotubes in biocompatible dispersants. J Nanopart Res 12(1):75-82

Raman V, Abbas A (2008) Experimental investigations on ultrasound mediated particle breakage. Ultrason Sonochem 15(1):55-64

Sager TM, Porter DW, Robinson VA, Lindsley WG, SchweglerBerry DE, Castranova V (2007) Improved method to disperse nanoparticles for in vitro and in vivo investigation of toxicity. Nanotoxicology 1:118-129

Shimoda K, Park J-S, Hinoki T, Kohyama A (2007) Influence of surface structure of $\mathrm{SiC}$ nano-sized powder analyzed by X-ray photoelectron spectroscopy on basic powder characteristics. Appl Surf Sci 253(24):9450-9456

Singh B, Jena J, Besra L, Bhattacharjee S (2007) Dispersion of nano-silicon carbide $(\mathrm{SiC})$ powder in aqueous suspensions. J Nanopart Res 9(5):797-806

Suslick KS, Price GJ (1999) Applications of ultrasound to materials chemistry. Annu Rev Mater Sci 29(1):295

Suslick KS, Didenko Y, Fang MM, Hyeon T, Kolbeck KJ, McNamara WB, Mdleleni MM, Wong M (1999) Acoustic cavitation and its chemical consequences. Philos Trans R Soc Lond A 357(1751):335-353. doi:10.1098/rsta.1999. 0330

Tartaj P, Reece M, Moya JS (1998) Electrokinetic behavior and stability of silicon carbide nanoparticulate dispersions. J Am Ceram Soc 81(2):389-394 
Taurozzi JS, Hackley VA, Wiesner MR (2011) Ultrasonic dispersion of nanoparticles for environmental, health and safety assessment-issues and recommendations. Nanotoxicology 5(4):711-729. doi:10.3109/17435390.2010. 528846

Teeguarden JG, Hinderliter PM, Orr G, Thrall BD, Pounds JG (2007) Particokinetics in vitro: dosimetry considerations for in vitro nanoparticle toxicology assessments. Toxicol Sci 97(2):614. doi:10.1093/toxsci/kfm097

Vankoningsloo S, Piret J-P, Saout C, Noel F, Mejia J, Zouboulis CC, Delhalle J, Lucas S, Toussaint O (2010) Cytotoxicity of multi-walled carbon nanotubes in three skin cellular models: effects of sonication, dispersive agents and corneous layer of reconstructed epidermis. Nanotoxicology 4(1):84-97. doi:10.3109/17435390903428869

Vauthier C, Cabane B, Labarre D (2008) How to concentrate nanoparticles and avoid aggregation? Eur J Pharm Biopharm 69(2):466-475

Yates PD, Franks GV, Jameson GJ (2008) Orthokinetic heteroaggregation with nanoparticles: effect of particle size ratio on aggregate properties. Colloid Surf A 326(1-2): 83-91 